and making them accessible for catalysis (Fig. 1b,c). The hydrolysis reaction can be followed by the emerging fluorescence of the hydrolysed FDP.

The process of masking and unmasking the enzymes is reversible, and thus bioactivation can be turned on or off on demand by applying more or less mechanical strain. When the film is no longer stretched, the enzymes are again masked by the dense polymer barrier and the catalysis is switched off.

The researchers identified two important requirements needed for successful mechanically tunable enzymatic reactions. First, the barrier should be thick enough to prevent enzyme diffusion through the unstretched barrier, but at the same time it should be relatively thin to unmask the enzyme on stretching. Second, the critical stretching degree required for the biocatalytic activation was found to be $70 \%$, a significant level of deformation.

The work of Voegel and co-workers shows that the design of 'cryptic-like' surfaces capable of reversibly inducing biochemical reactions by applying a mechanical stress is feasible with layer-bylayer materials. Manipulating the construct design, for example, by varying the barrier thickness and reservoir parameters, is a way to tune the amplitude of the biocatalytic activity triggered by mechanical stimuli. The work has great potential in the design of responsive bioengineered materials aimed at triggering and modulating chemical and biochemical reactions by mechanical action, with applications ranging from smart microbiofluidic devices to mechanically controlled biopatches.
Eugenia Kharlampieva and Vladimir V. Tsukruk are at the School of Materials Science and Engineering, Georgia Institute of Technology, Atlanta,

Georgia 30332, USA.

e-mail:vladimir@mse.gatech.edu

References

1. Luzinov, I., Minko, S. \& Tsukruk, V. V. Prog. Polym. Sci. 29, 635-698 (2004).

2. Mertz, D. et al. Nature Mater. 8, 731-735 (2009).

3. Decher, G. \& Schlenoff, J. B. (eds) Multilayer Thin Films: Sequential Assembly of Nanocomposite Materials (Wiley-VCH, 2003).

4. Jiang, C. \& Tsukruk, V. V. Adv. Mater. 18, 829-840 (2006).

5. Srivastava, S. \& Kotov, N. Acc. Chem. Res. 41, 1831-1841 (2008).

6. Hiller, J., Mendelsohn, J. D. \& Rubner, M. F. Nature Mater. 1, 59-63 (2002).

Lvov, Y. \& Caruso, F. Anal. Chem. 73, 4212-4217 (2001).

8. Kharlampieva, E. \& Sukhishvili, S. J. Macromol. Sci.-Polym. Rev. 46, 377-395 (2006).

9. Sukhorukov, G. B. \& Möhwald, H. Trends Biotechnol. 25, 93-98 (2007).

10. Genzer, J. \& Efimenko, K. Science 290, 2130-2133 (2000).

11. Betanzos, M., Chiang, C. S., Guy, H. R. \& Sukharev, S. Nature Struct. Biol. 9, 704-710 (2002).

\title{
RELAXING THE VACUUM
}

There are few topics in physics more prone to misrepresentation than the Casimir force. In popular discourse, the term is commonly preceded by 'ghostly', as though there is something barely credible about the manifestation of an attractive interaction between two surfaces separated by a vacuum. Interpretations in terms of virtual particles or suppressed quantum fluctuations of the electromagnetic field only encourage that view. But regarded as the familiar dispersion force resulting from induced dipoles, 'slowed down' by the finite speed of photons, the Casimir force becomes altogether more prosaic.

All the same, proposals to alter its influence - to engineer it have about them something of the marvellous, as though the inescapable exigencies of nature are somehow being cheated. This possibility, however, was already implicit in Evgeny Lifshitz's recasting of the Casimir force in 1956, when he worked out the theory for real materials with finite dielectric permittivity (that's to say, finite conductivity). It's easy to see from Lifshitz's theory that, for certain choices of plate materials and media separating them, the Casimir force can actually be made repulsive. All the same, it wasn't until earlier this year that the right combination of materials - silica, gold and an organic liquid - was found ${ }^{1}$. (It's often overlooked that a classical analogue of this repulsive force, due to density fluctuations of a fluid at its critical point between two surfaces, was seen some time ago in superfluid helium ${ }^{2}$.)

This raises the prospect of 'quantum levitation' and of ultralow friction and contactless bearings for microand nanoelectromechanical systems (MEMS and NEMS, respectively). But the reality is trickier. The choice of materials, for example, is commonly dictated by other engineering considerations. Transparent dielectric surfaces such as silica will in themselves reduce the Casimir attraction relative to reflective metals, even if they don't alter its sign. But they also have a tendency to accumulate surface charges in air, which, on nonconductive media, cannot be dissipated and create a strong electrostatic attraction. A thin film of noble metal such as gold will allay that issue, but at the expense of constraining the dielectric function and leaving little scope for tuning the Casimir force.

Davide Iannuzzi and colleagues at the University of Amsterdam have now shown that it is possible to combine the best of both worlds ${ }^{3}$. Conductive transparent metal oxides such as indium tin oxide (ITO), indispensable for semiconductor display technology, offer amenable dielectric properties

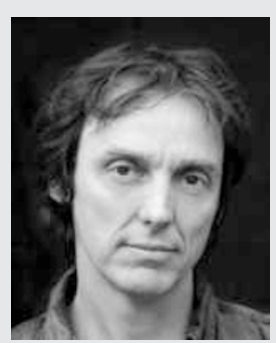

PHILIP BALL

while dispersing surface charges in air. The researchers have used a customized atomic-force microscope to measure the force between a gold-coated polystyrene microbead and a flat surface coated with gold or ITO. In both cases, the Casimir force clearly dominates over any residual Coulombic force in ambient conditions for separations down to about $60 \mathrm{~nm}$. But for ITO the attractive force is about a factor of two smaller. This, they say, should leave plenty of scope for tailoring the interaction in MEMS/ NEMS applications. It's an intriguing example of how the right choice of materials can alter the basic physics. $\square$

\footnotetext{
References

1. Munday, J. N., Capasso, F. \& Parsegian, V. A. Nature 457, 170-173 (2009).

2. Garcia, R. \& Chan, M. H. W. Phys. Rev. Lett. 88, 086101 (2002)

3. de Man, S., Heeck, K., Wijngaarden, R. J. \& Iannuzzi, D. Phys. Rev. Lett. 103, 040402 (2009).
} 